\title{
Nutrición relacional: configuración de identidades como red sistémica entre familia e instituciones educativas en primera infancia
}

\section{Relational nutrition: configuration of identities as a systemic network between families and educational institutions in early childhood}

\author{
Camila del Pilar Díaz Carranza ${ }^{1}$ \\ Mónica Paola Medina García ${ }^{2}$ \\ Carolina Sánchez Güiza ${ }^{3}$ \\ DOI: 10.29151/hojasyhablas.n17a2
}

\section{Resumen}

Si un sistema es un conjunto de elementos en interacción, según la teoría de Bertalanffy, establecer una analogía con la educación en los primeros años de vida del ser humano implica reconocer a la familia e instituciones educativas de atención a la primera infancia como parte indispensable de los microsistemas que nutren y configuran identidades en cada uno de los individuos inmersos en esa red sistémica. El presente artículo de reflexión surge de la investigación en curso denominada Nutrición relacional desde el ámbito familiar e institucional en primera infancia de la Unimonserrate y está organizado en cuatro apartados: el primero es una introducción de acuerdo con la problemática alrededor de las formas de violencia que se evidencian en nuestra sociedad. El segundo, que se denomina enfoque sistémico: características y relaciones con la configuración de identidades en primera infancia, define las relaciones y concepciones de lo sistémico, individuos e identidades. El tercer apartado, abarca los argumentos relacionados con los aportes nutricionales desde el contexto familiar. Posteriormente, el cuarto apartado menciona los aportes desde las instituciones educativas de primera infancia.

Palabras clave: Familia; Nutrición Relacional; Primera Infancia; Enfoque Sistémico; Identidad; Educación.
If a system is a set of interacting elements, according to Bertalanffy's theory, establishing an analogy with education in the first years of the human being's life implies recognizing the family and educational institutions from early childhood as an indispensable part of the microsystems that nurture and configure identities in each person immersed in that systemic network. The present reflection paper is part of the Unimonserrate research "Relational nutrition from the family and institutional environment in early childhood" and is organized into four sections. The first is an introduction about evident violence in our society. The second is called systemic approach: characteristics and relationships with the configuration of identities in early childhood and defines the relationships and conceptions of the systemic, individuals and identities. The third section covers the arguments related to nutritional contributions from the family context. Subsequently, the fourth section mentions the contributions from early childhood educational institutions.

Keywords: Family; Relational Nutrition; Early Childhood; Systemic Approach; Identity; Education.

\footnotetext{
${ }^{1}$ Magistra en Familia, Educación y Desarrollo de la Unimonserrate. Correo: cddiaz@unimonserrate.edu.co ORCID: https://orcid.org/00000002-5548-1775

${ }^{2}$ Magistra en Familia, Educación y Desarrollo de la Unimonserrate. Correo: mpmedina@unimonserrate.edu.co ORCID: https://orcid. org/0000-0002-0365-5219

${ }^{3}$ Magistra en Familia, Educación y Desarrollo de la Unimonserrate. Correo: csanchezg@unimonserrate.edu.co ORCID: https://orcid. org/0000-0001-7024-3356
} 


\section{Introducción}

En Colombia, de acuerdo con el Informe Forensis de Vélez (2015), el 77\% de los casos de violencia contra niños, niñas y adolescentes fueron producidos por sus propios familiares, entre los que se cuentan a padres, madres, padrastros, madrastras, hermanos, tíos, abuelos y otros familiares no consanguíneos. Así mismo, el 64\% de los casos se presentaron en la vivienda, como principal escenario de agresión para los niños y las niñas.

Para el contexto de Bogotá, y según cifras de la Personería de Bogotá, en el año 2018 las Comisarías de Familia y el Instituto Colombiano de Bienestar Familiar (ICBF) atendieron cerca de 13.957 casos de violencia intrafamiliar en la ciudad, lo que indica que diariamente se atienden al menos 66 hechos de acoso y maltrato al interior de los hogares. De lo anterior, es posible identificar que estas formas de violencia explícita hacen parte de una problemática cultural que se ha gestado y tiene raíces históricas que son indispensables reconocer, con el fin de transformar el presente y futuro de la sociedad, donde los lugares que se piensan seguros, en realidad, son los más riesgosos y vulnerables debido a las formas violentas de relación que emergen, se naturalizan y permanecen.

La violencia que ha dejado nuestra historia se ve representada en nuevas formas que permanecen en diferentes ámbitos como la familia, la escuela, el barrio, la comunidad, entre otros, como lo refiere Martínez (2013) en su propuesta de no violencia, desde la cual sostiene que parte de la crisis de nuestra sociedad se sustenta en el "imagi- nario atá $\neg$ vico que legitima la violencia como método para dominar y destruir el mal” (p. 33)

Por otra parte, pero de la mano, las relaciones entre adultos y niños son estrategias de supervivencia mediadas por relaciones de poder, donde quien define las normas y las formas de interacción y resolución de conflictos en los primeros años de vida es el adulto, principalmente, a partir de mecanismos como la represión e imposición, lo que, de cierto modo invisibiliza la consciencia y reflexión conjunta, siendo el espejo de una sociedad que trasciende en una cultura impuesta por infinitas circunstancias.

En este sentido, las formas culturales heredadas permean la sociedad y se observan desde las prácticas profesionales en instituciones educativas, en las cuales las familias y maestros pueden perpetuar prácticas de violencia adquiridas inconscientemente. Se evidencian algunas dificultades en el ámbito socio afectivo de los niños y las niñas, en sus relaciones y reacciones compulsivas de agresión desde los primeros años de vida o situaciones de multiplicidad de violencias al interior de las familias.

Por lo anterior, comprender las prácticas de cuidado y nutrición relacional ${ }^{4}$ permite hacer una lectura desde un enfoque sistémico de las relaciones e interacciones que, desde la primera infancia se hacen presentes, se reflejan en las formas de relación de los niños y las niñas en sus primeros años de vida, además de configurar nuevas identidades, formas de encontrarse y relacionarse con las otras personas.

4 La categoría de prácticas de cuidado y nutrición relacional hace referencia a las formas de cuidado, crianza y amor que en las familias y escuelas de atención a la primera infancia que se visibilizan bajo el concepto de Nutrición relacional propuesto por Linares (2012), en tanto, el amor complejo que todo individuo requiere desde componentes emocionales, cognitivos y pragmáticos. 
Nutrición relacional: configuración de identidades como red sistémica entre familia e instituciones educativas en primera infancia.

Cada ámbito y microsistema deja ver un mundo de relaciones importantes de comprender en las que la nutrición relacional, como lo plantea Linares (1996), es el concepto principal de análisis, el cual permite entablar conexiones entre las familias e instituciones educativas que atienden a la primera infancia. De esta manera, reflexionar alrededor de las formas y acciones que se brindan a niños y niñas, en torno del cuidado de sí mismos $y$ de las relaciones que entabla con otros, son parte fundamental para el resto de la vida.

El concepto de la nutrición relacional ha sido trabajando e investigando principalmente en España, a través de estudios de pilotaje en instituciones educativas con adolescentes, debido a la preocupación por fenómenos disruptivos que se producen en el marco educativo. Dichos estudios permiten un análisis de la emergencia de la nutrición relacional en los vínculos entre profesores y estudiantes, para así mismo promover procesos de enseñanza y aprendizaje significativos.

En esta misma línea, Ecuador realizó una investigación en la Universidad Católica, donde se refiere a las relaciones nutricionales de un sistema familiar que depende básicamente de los roles y normas que se asignen dentro del hogar para cada uno de sus miembros, esto permitió que cada uno pueda tener suficiente autonomía para tomar decisiones acordes a la edad en la que se encuentra y de esta manera hacer una construcción socioemocional, que les permitiera sobrevivir, subsistir $y$ adaptarse a su entorno.

Otras investigaciones en Bogotá, describen fenómenos relacionales en el caso de padres separados con sus hijos adolescentes o el embarazo de adolescentes, los cuales abordan de manera holística una red de relaciones y situaciones que desde un enfoque sistémico complejo permiten comprenderlas. La Secretaría de Salud de Bogotá (2018) realizó un estudio sobre el significado del embarazo y el sistema relacional de la familia, en el que se concluye que durante el embarazo se lleva a cabo el proceso emocional más importante para el niño, en el que influye evidentemente la familia y quienes rodean al bebé para capacitar física, moral y cognitivamente al nuevo miembro de la familia, para que en un futuro éste pueda ser capaz de desenvolverse de forma asertiva a nivel social.

En este orden de ideas, las tendencias en las investigaciones muestran que las relaciones entre los sistemas familiares influyen directamente en la formación de la dimensión socio-afectiva de sus hijos. Desde esta perspectiva, permitir una mirada reflexiva alrededor de la nutrición relacional en las familias

e instituciones educativas en los primeros años de vida, es parte de comprender cómo estas relaciones e interacciones posibilitan la configuración de identidades.

\section{El enfoque sistémico: Características y rela- ciones con la configuración de identidades en primera infancia.}

En este apartado, el análisis del enfoque sistémico es fundamental para incluir la nutrición relacional en los contextos en donde transita la infancia actualmente (escuelas de primera infancia o jardines) y como parte indispensable para la configuración de identidades y transformación de formas de violencia a formas de relación que nutran al individuo.

Bertalanffy en 1969 formuló la teoría general de sistemas, que, aunque surge de un modelo biológico denominado "organicista", que conceptualizaba a los organismos como sistemas abiertos en constante interacción, el mismo autor en 1976 lo 
transformó en un concepto más humanista para la percepción de la naturaleza humana y sus relaciones. Este nuevo enfoque influyó en la psicología para el estudio de las realidades, la comunicación y las estructuras, empezando a entender su complejidad.

Este intento por comprender las relaciones humanas como sistemas, introdujo otra forma de interpretación del mundo. Así pues, la totalidad, según Bertalanffy (1976) "es un conjunto de elementos en interacción" (p.37), se caracterizan porque cualquier cambio en alguna de las partes afecta a los demás y ocasionalmente al sistema (sinergia) y sin embargo tiende a conservar la identidad (entropía).

Acorde a lo anteriormente señalado, cada individuo podría ser un sistema, y cada espacio en el que se encuentra dicho sistema se considera como un supra sistema: la familia, por ser el medio en el que se está inmerso en los primeros años de vida, $y$ en la actualidad, las instituciones educativas para la primera infancia son otro medio de interacción en los que transita la infancia. Son, de esta manera, un sistema abierto y autopoiético por las relaciones constantes que mantiene con el medio en el que se encuentra inmerso:

Una maquina autopoiética es una máquina organizada como un sistema de procesos de producción de componentes concatenados de tal manera que producen componentes que: 1) generan los procesos (relaciones) de producción que los producen a través de sus continuas interacciones y transformaciones, y 2) constituyen a la máquina como una unidad en el espacio físico (Maturana, 2004, p. 69)

Siguiendo lo anterior, estas relaciones e interacciones transforman y, por tanto, son consti- tutivos en la construcción del ser, son parte de las experiencias de vida, de las narrativas y las relaciones que se generan desde allí. Desde la gestación y durante los primeros años de vida, el ser humano se encuentra inmerso en un primer sistema familiar y en un sistema educativo. Son medios, que hacen parte de la comprensión y transformación de identidades. Esta totalidad, comprende también todo un tejido social, cultural, político y económico como componentes interactuantes con el individuo desde sus primeros años de vida.

Por otra parte, Bourdieu habla de un espacio social en el que existen distintos tipos de capital: económico, social, cultural, relacionados con campos de poder que constituyen desde la infancia a los seres humanos, conformando un habitus como:

Sistemas de disposiciones duraderas y transferibles, estructuras estructuradas predispuestas a funcionar como estructuras estructurantes, es decir, como principios generadores y organizadores de prácticas y de representaciones que pueden ser objetivamente adaptadas a su meta sin suponer el propósito consciente de ciertos fines ni el dominio expreso de las operaciones necesarias para alcanzarlos (Bourdieu, 2007, p. 86).

Es decir, el habitus funciona como estructura que se transfiere desde lo social y genera prácticas sociales que se determinan como constitutivas del ser humano. En este sentido, cada espacio en el que transita la infancia es una estructura estructurante o un componente esencial que constituye a cada individuo y lo configura de acuerdo a las experiencias, situaciones, relaciones e interacciones que genera.

Las relaciones sociales y las relaciones que se tejen con los sistemas externos tienen que ver con 
Nutrición relacional: configuración de identidades como red sistémica entre familia e instituciones educativas en primera infancia.

el componente cultural que proporciona significados, ellas nutren las construcciones de cada individuo, pues "el ser humano es a la vez un ser cultural y social, en todo lo que hace, piensa y quiere" (Sacristán, 2010, p.110). Es un ser que, además, se encuentra en un sistema más amplio, en un ambiente que, desde la ecología profunda, propone Capra (1996) "reconoce la interdependencia fundamental entre todos los fenómenos y el hecho de que, como individuos y como sociedades, estamos todos inmersos en los procesos cíclicos de la naturaleza" (p. 28), es decir somos parte de un mundo natural que depende de todos y es para todos.

Todas estas relaciones e interacciones que se pueden establecer conforman un legado importante y hacen parte de una narrativa, que como la describe Linares (2012) "es algo que el ser humano hace ininterrumpidamente a lo largo de su existencia, en un proceso de complejidad progresiva, desde la vida intrauterina hasta bien avanzada la edad adulta" (p. 96) por lo tanto, dota de significados al individuo, aquél que va configurando una narrativa, en donde, sin duda el significado de la experiencia relacional es enorme.

Plantear el concepto de identidad implica entonces, definirlo desde un enfoque sistémico, lo que requiere recordar a Bateson (1956) alrededor de un concepto importante denominado cismogénesis que es "un proceso de diferenciación en las normas de la conducta individual resultante de la interacción acumulativa entre los individuos" (p. 98), por lo tanto, las interacciones y narrativas que emergen a partir de cada legado relacional van constituyendo identidades.

En este orden de ideas, la identidad "es un producto narrativo especializado" que se configura a lo largo de la vida y es dirigida por la nutrición relacional: "vivencia de ser complejamente ama- do" como un proceso decisivo para la constitución de la personalidad (Linares, 2012, p. 98), en tanto, la identidad pertenece a una dimensión personal del ser humano que se enriquece con las experiencias relacionales que se tejen en la vida.

Así mismo, aquellas relaciones que se tejen a lo largo de las interacciones en las relaciones de vida desde la gestación se encuentran definidas por un contexto cultural que constituye la personalidad como aquella "dimensión individual de la experiencia relacional acumulada, en diálogo entre pasado y presente, y encuadrada por un sustrato biológico y por un contexto cultural" (Linares, 2012, p. 94). De esta manera, el pasado forma parte de una historia importante, pero además sujeta a transformarse desde los diálogos que se puedan emplear en el presente, en una nueva narrativa sujeta por la nutrición relacional que se posibilite para cada individuo.

Desde esta mirada, se sustenta la función decisiva de la familia, pero además de la educación en la configuración de identidades que, desde la primera infancia, específicamente desde la gestación, empiezan a ser determinantes y se accionan en los diferentes contextos en los que se encuentran niños y niñas.

Es así como, involucrar en la familia e instituciones educativas de primera infancia, experiencias, relaciones e interacciones amorosas que se vivan en la cotidianidad, teniendo en cuenta que, un individuo depende del amor que recibe, pero al mismo tiempo de cómo lo perciba, supone reconocer a un individuo como ser histórico-social-relacional que confluye en un contexto que lo configura y una cultura que lo constituye.

Nuestra realidad cultural determina una tendencia a la competitividad, el individualismo y 
las formas de violencia que se han transferido y se deben comprender para actuar sobre la educación inicial, la familia e instituciones educativas, trabajando alrededor de las prácticas que nutren la conformación de individuos para una sociedad libre de violencia. Según Sacristán (2010), la cultura "nos proporciona un sentido del 'nosotros' como miembros de un grupo social que tiene una trayectoria histórica; nos da conciencia de ser continuadores de 'otros', pero semejantes a 'nosotros' ( $\mathrm{p}$. 104). Reconocer esto, nos permite tener empatía y desde allí generar un reconocimiento del otro.

\section{La nutrición relacional: componente esen- cial para la comprensión en la configuración de identidades en el ámbito familiar.}

El ámbito familiar es uno de los microsistemas indispensables en los que la infancia se configura y construye las primeras relaciones, comprende aspectos culturales y sociales que hacen parte de una comunidad en la que se encuentra inmerso. A partir del enfoque sistémico, se define a la familia como un "conjunto organizado e interdependiente de personas en constante interacción, que se regula por unas reglas y por funciones dinámicas que existen entre sí y con el exterior" (Espinal, Gimeno y González, 2000, p. 2). En este sentido, la familia es entendida como un grupo con una identidad propia y como escenario en el que tiene lugar un amplio entramado de relaciones.

Según Bronfenbrenner (1986) la familia es el microsistema más importante porque configura en mayor medida el desarrollo de la persona desde su concepción. La familia, como una totalidad, se construye mediante un sistema de valores y creencias compartidos por las experiencias vividas a lo largo de la vida, y por los rituales y costumbres que se transmiten generacionalmente, podríamos comprender que existen varios tipos de familia y estructuras familiares.

Para Hernández (1997), "la familia puede definirse desde cuatro dimensiones: institución social, grupo, constructo cultural y conjunto de relaciones emocionales". De lo anterior, podríamos interpretar que la familia es un sistema que se ve permeado por elementos culturales, sociales, emocionales (valores, creencias), organizacionales (límites, normas) y un conjunto de identidades y roles, que van determinando dinámicas relacionales complejas que se evidencian, a través, de las prácticas de amor (reconocimiento, validación y afecto) y cuidado que se tejen dentro de ella.

Se presume que la familia tiene prácticas de cuidado que pueden ser visibles en la convivencia, en las relaciones de reconocimiento, validación o invalidación de los integrantes (conyugal o parento-filial), por lo anterior, se construyen narrativas dependiendo del lugar en el que se posiciona el otro y que pueden ser influenciadas por el mito familiar o pautas que pueden ser legado de generación en generación, un ejemplo de ello puede ser "la letra con sangre entra" (Cervantes, 1615, cap. XXI) refiriéndose a un castigo generalmente físico para educar o corregir. Desde esta mirada sistémica de la familia, la relación con un concepto sistémico relacional como la nutrición relacional permite generar reflexiones interesantes para comprender la importancia de las relaciones que allí se tejen.

El concepto de nutrición relacional se desarrolla inicialmente por Linares (2012) quien es psiquiatra por la Universidad de Granada, psicólogo por la Universidad de Barcelona, doctor en medicina por la Universidad Autónoma de Barcelona y terapeuta sistémico español, quien denomina la nutrición relacional como la conciencia de ser complejamente amado, por lo tanto, el amor 
Nutrición relacional: configuración de identidades como red sistémica entre familia e instituciones educativas en primera infancia.

es un fenómeno relacional complejo que incorpora elementos cognitivos y pragmáticos a los componentes emocionales. Este sistema de relaciones basadas en el amor configura la personalidad de cada individuo desde la "vivencia subjetiva de ser amado" (p. 28). La nutrición relacional es una de las bases conceptuales para comprender los procesos educativos de los individuos de manera sistémica. En esa medida, las relaciones que se enmarcan desde la gestación y los primeros años de vida con padres y cuidadores incluyen un pensar, sentir y hacer amoroso, que se configura en procesos relacionalmente nutricios para la formación y crecimiento de cada ser humano.

Desde esta mirada, es indispensable interpretar los tres componentes que plantea Linares (2012). En el aspecto cognitivo, se encuentra un concepto fundamental que es el reconocimiento, "como aquella necesidad de verse como un individuo con capacidades y características propias, validado desde su sentir, necesidades y habilidades, en el caso de los padres hacia sus hijos se determina como la capacidad para reconocerlos y valorarlos adecuadamente, que en algunos casos puede ser una descalificación o fracaso de la valoración de las cualidades personales por parte de figuras relevantes del entorno relacional" (Linares, 2012, p. 30).

En este aspecto, se establece una relación con las concepciones de infancia de los padres de familia, en la medida en la que contemplan al individuo niño o niña como un ser con gran potencial y por tanto validan su sentir como parte del reconocer que hace parte de un contexto. Por otro lado, desde una mirada como profesionales investigadores en contextos educativos y de trabajo con familias, se tiende a observar la poca validación social de la infancia, al mantener creencias en las cuales el entendimiento hace parte de etapas pos- teriores, por lo tanto, la invalidación y descalificación dan forma a aquella concepción de infantes como personas con menor agencia para la toma de decisiones.

En relación con los componentes pragmáticos, que hacen referencia a las prácticas y acciones amorosas concretas durante la socialización, involucrando aspectos como la protección y normatividad. Es dentro de estas prácticas que se configuran nuevas identidades, que permiten que se demuestre o no el amor como fenómeno relacional complejo desde lo pragmático (hacer), lo cognitivo (pensar) y emocional (sentir). Esto supone la materialización de las creencias o concepciones de las familias en su accionar, lo cual puede ser coherente o no. Aunque se concibe al niño como un ser con capacidades y habilidades para involucrarse en un grupo, sin embargo, perdura la preocupación e inseguridad por reconocerlo. O se concibe el amor desde la cercanía, cuidados, afectos $\mathrm{y}$ amor que pueden en lo pragmático ser impedidos por las dinámicas de trabajo y desvinculación afectiva.

Otra de las prácticas en las familias se genera a partir de lo normativo en las cuales permanecen formas de premio-castigo que develan formas de violencia que se transmiten consciente o inconscientemente, en las cuales, la consciencia de cada acción desde lo real se invisibiliza y no permite un pensamiento crítico y reflexivo frente a las propias acciones, sino la adquisición de la norma de carácter represivo o como una forma de poder dentro de una lucha de poderes.

La desnutrición relacional de las familias que podrían estructurar formas de violencia se constituye al concebir la figura del hijo parentalizado, el abandono físico y/o emocional de uno de los padres, la brecha generacional, pautas relacio- 
nales sobreprotectoras, ausencia de norma, crítica o reflexión ante ella o diferencias relacionales evidentes entre géneros, entre otras. Todo lo anterior configura identidades, en donde es posible normalizar algunas formas de violencia que van construyendo una narrativa en el individuo.

Con lo anterior, también cobra importancia la influencia del sistema externo o macro sistemas que confluyen en el sistema familiar e individual, como las extensas jornadas laborales, el cuidado de los niños y las niñas a los abuelos o nanas, el uso constante de aparatos tecnológicos, el aislamiento que facilita la entropía y por tanto el deterioro del sistema.

Finalmente, el componente emocional supone el cariño y la ternura como los elementos más importantes en el contexto parento-filial, son sentimientos de entrega y disponibilidad para el otro, por quien se haría cualquier cosa. Cabe afirmar que el cariño y la ternura son los componentes del amor complejo más vulnerables en el corto plazo, pero más resistentes en el largo: se afectan fácilmente, pero no les cuesta recuperarse (Linares, 2012)

Las interacciones en el ámbito familiar para brindar un ambiente nutricional requieren de componentes de cariño y ternura que son subjetivos de acuerdo a las características de cada familia, pero necesarios para construir esa consciencia de ser amado. En este sentido, Linares (2012) afirma que "la familia de origen es, el sistema relacional de mayor relevancia en lo que a la construcción de la personalidad se refiere, puesto que en ella se producen los intercambios más influyentes sobre el desarrollo narrativo e identitario" (p. 102).

La familia es para el niño su primer núcleo de convivencia y de actuación, donde irá mode- lando su construcción como persona a partir de las relaciones que allí establezca y, de forma particular, según sean atendidas sus necesidades básicas (Brazelton y Greenspan, 2005). Este proceso de construcción de su identidad se dará dentro de un entramado de expectativas y deseos que corresponderán al estilo propio de cada núcleo familiar y social.

Es entonces, como las interacciones y relaciones en la familia aportan y nutren la configuración de identidades en los niños y las niñas desde la primera infancia, pues como mencionan Espinal, Gimeno y González (2000), la familia permanece en contacto entre sí a partir, "de una serie continua de intercambios que suponen una mutua influencia y no una mera causalidad lineal, sino bidireccional o circular, que tiende a mantenerse estable" (p. 5). En este sistema se encuentran unos subsistemas importantes que se especifican a continuación.

\section{Subsistemas conyugal y parental: roles decisivos}

El entorno familiar del niño está conformado por dos dimensiones relacionales: la conyugalidad y la parentalidad. En la familia estos dos subsistemas se consolidan como indispensables dentro de las dinámicas familiares y cada uno tiene una función específica y un rol nutricional relacional, que permite a los hijos configurar su identidad con las interacciones entre todos los miembros de la familia, pero además a la organización y funcionamiento de los subsistemas.

Cada uno tiene características específicas que plantea Linares (2006) dentro de cinco axiomas; en el primero hace referencia a la dimensión individual de cada subsistema, pero a la vez su funcionamiento varía de acuerdo a factores cul- 
Nutrición relacional: configuración de identidades como red sistémica entre familia e instituciones educativas en primera infancia.

turales, como por ejemplo cuando recae más sobre la mujer la función parental. Lo anterior, puede llevar a un proceso de reflexión, en el cual es determinante comprender el contexto cultural y concepciones de la pareja para analizar los vínculos relacionales nutricios que se están edificando en cada subsistema.

En el segundo axioma, Linares (2006) deja ver el papel fundamental que tiene en la relación la historia que cada miembro de la pareja tiene con sus familias de origen y por tanto cómo esta historia permea la construcción y el camino presente. En el tercer y cuarto axioma, nombra esa relación estrecha que tienen estos dos subsistemas desde parámetros complejos, en la que estas dos son independientes, pero al mismo tiempo dependientes la una de la otra.

En este sentido, estas dos dimensiones son interdependientes, porque cada una actúa como un pequeño sistema que se debe fortalecer constantemente desde las relaciones e interacciones que se tejan entre la pareja, los padres con los hijos, pero a la vez son dependientes, porque se necesitan la una de la otra para mantener una armonía en el sistema familiar que influye, además, en la construcción de identidad de los individuos y de estas formas de relación, dependiendo si son formas de represión, castigos, descalificaciones, ausencias o por el contrario cariño, diálogo, reflexión y validación, que puedan transformar la historia de una sociedad.

El quinto y último axioma, menciona el componente eco-sistémico en cada subsistema que "está sometido a la evolución del ciclo vital y al influjo de los más variados factores ambientales" (p. 75), es decir, su comprensión requiere una mirada holística, cultural, política, histórica y social que permea a los individuos y configura for- mas de relación.

En el subsistema conyugal Linares (2006) describe que:

Se fundamenta en una reciprocidad cognitiva, emocional y pragmática, mediante la cual ambos miembros negocian un acuerdo que implica un pensar amoroso (reconocimiento y valoración), un sentir amoroso (ternura y cariño) y un hacer amoroso (deseo y sexo, principalmente). Todo ello exige el intercambio, es decir, un ejercicio de dar y recibir de forma equilibrada, con un importante componente igualitario (p. 386).

Por consiguiente, comprender los vínculos relacionales nutricios desde un pensar, sentir y hacer amorosos hacia los niños y las niñas, supone reconocer la configuración del subsistema conyugal, es decir, conocer las relaciones simétricas o complementarias de la pareja que influyen en la configuración de identidad de los hijos. La conyugalidad, se contrapone a la relación que se espera en la parentalidad, pues es una relación desigual en la que dar y recibir no pueden estar equilibrados, sin embargo, también asume componentes cognitivos, emocionales y pragmáticos, y este último tiene grandes diferencias, pues según Linares (2007),

El hacer amoroso parental consiste, fundamentalmente, en el ejercicio de la sociabilización. Ésta no es otra cosa que una preparación adecuada para integrarse en la sociedad, y se compone de dos integrantes de igual importancia: la normatividad, que debe garantizar el respeto de la sociedad por el individuo, y la protección, encargada de que ese respeto sea recíproco (p. 385). 
En este orden de ideas, es del rol parental involucrar a los hijos en una sociedad y esto implica una preparación para la vida en comunidad que requiere de unas normas iniciales que se generan en casa y un ejercicio de autoridad. Linares (1996) plantea que una de las características esenciales en esta relación es la circularidad, pues ella:

Preside las funciones parentales nutricias, mediante las cuales los padres suministran a sus hijos nutrición emocional a través de circuitos paralelos e interconectados que tienen que ver con el reconocimiento, el amor y la valoración. Sintiéndose queridos, reconocidos y valorados los niños son capaces de integrar normas y seguridad en proyectos personales coherentes. Además, comunican a los padres que ellos también son valorados y queridos, lo cual les permite nutrirse nutriendo (p. 76).

Por ende, entender las relaciones como un sistema que es circular y se alimenta constantemente, es reconocer que los vínculos son recíprocos y complementarios, por ejemplo, la forma en que un niño o una niña resuelve un conflicto puede poner en evidencia las prácticas de nutrición relacional y cuidado que se están constituyendo dentro del hogar.

La conyugalidad y la parentalidad actúan dentro de un sistema familiar autopoiético y dependiente de un intercambio constante y constituyente de identidades. Es decir, no es una relación lineal sino bidireccional en la cual todos son nutridos y así mismo configurados en su identidad, lo que permite una circularidad que al mismo tiempo crea transformación al continuar generación tras generación y trascribir una historia familiar y social.
En estas dinámicas familiares, la personalidad individual se forja en contacto con las dos dimensiones mencionadas, a través, de "su organización (cohesión, adaptabilidad y jerarquía características) y mitología (valores y creencias, clima emocional y rituales propios) en procesos regidos por la nutrición relacional" (Linares, 2012, p. 107).

Dichos procesos de nutrición relacional inician desde las narrativas que el individuo emprende desde la gestación, los componentes pragmáticos y emocionales que se generen desde allí, "el bebé, y el niño sin duda, de forma arrolladora son auténticas máquinas narrativas, que construyen infinitas historias de ternura, de soledad, de alegría, de miedo, de consuelo y de tantos matices como se nos pueda ocurrir" (Linares, 2012, p. 97), referente a ello este sistema familiar genera unas reacciones que ponen en juego los componentes cognitivos, pragmáticos y emocionales frente a la narrativa relacional del individuo.

\section{Nutrición relacional: concepto sistémico bá- sico en la educación inicial para la configu- ración de identidades}

A continuación, se construye un análisis desde otro microsistema indispensable en el cuál transita la infancia en la sociedad contemporánea: las instituciones educativas de primera infancia, que conforman un espacio esencial en el que niños y niñas pueden entablar relaciones nutricias que configuren identidades en ese flujo bidireccional familia, comunidad, equipo educativo.

La nutrición relacional en el ámbito educativo implica aquellas formas de relación que nutren y promueven el desarrollo socio-afectivo entre maestro y estudiantes, pero además involucra a todo un equipo educativo presente en un espacio que hacen parte de una cotidianidad que también 
Nutrición relacional: configuración de identidades como red sistémica entre familia e instituciones educativas en primera infancia.

configura identidades, pues la educación en primera infancia es un sistema importante que está presente en lo cotidiano de los niños y las niñas hoy, constituye relaciones e interacciones en la experiencia de vida que van conformando parte importante en la narrativa de cada individuo y por tanto de las relaciones que generan y acumulan en este otro sistema del que hace parte.

En este sistema educativo, en el cual la institucionalidad hace parte en la construcción de saberes, desde un enfoque sistémico se puede visualizar cómo un sistema complejo con múltiples elementos en relación, abierto al medio, y compensados homeostáticos ${ }^{6}$ entre sus elementos, los cuales son tres tipos: humanos, materiales y conceptuales o simbólicos. Entre estos elementos, se establecen relaciones informativas de las que podemos distinguir los siguientes tipos:

A. informaciones que se establecen el paradigma normativo o axiológico. B. informaciones para desarrollar la operatividad del sistema (legislación, planes, programas, presupuestos, objetivos...). C. informaciones para la acción educativa (métodos, asignación de recursos, materiales, coordinación...). D. informaciones para implementar la ejecución educativa (procedimientos, instrucciones, evaluaciones...) (Ballester, 2012, p. 21).

Todos estos procesos dentro de la escuela constituyen un tejido sistémico en constante interacción, que requiere de reflexión por parte del equipo de educadores, en pro de los aportes nutri- cios que se generan a niños y niñas en la configuración de identidades, que aludan a las necesidades sociales en las cuales se encuentra la emergencia por la solidaridad, formación de pensamiento crítico, la conciencia social, ética y política.

Así, es importante que converjan todos los aspectos requeridos para un desarrollo integral desde la primera infancia: las relaciones sociales, aquellos vínculos afectivos que van más allá de las palabras descriptivas, las narrativas, cada experiencia e interacción con otro, que constituye y configura identidades en la escuela. En palabras de Loris Malaguzzi (2011):

La escuela es, en realidad, un organismo vivo, un sistema. Decir que es un sistema, significa poner el acento sobre el dinamismo y la constructividad de las relaciones e interacciones entre pares, de manera que cada parte actúa, en el organismo como totalidad y viceversa (p. 52).

Por ende, se supedita la identidad al ser social, el que se configura con el otro y es permeado tanto cognitivamente como emocionalmente, logrando generar relaciones que dependen de la cercanía (familia-escuela) siendo algunas más complejas en vínculos que otras.

De este modo, la nutrición relacional en la escuela ya no depende de la combinación e interrelación de conyugalidad y parentalidad, pero sí de dimensiones relacionales que reflejan diferentes universos de vínculos, horizontales y verticales. "Por ejemplo, en la escuela el niño vivirá

\footnotetext{
5 "Cannon, hace más de cincuenta años, inventó la homeostasis y transformó al organismo en una red de procesos, dándonos la segunda visión fisiológica de su autonomía” (Maturana, 1992, p. 118). Por ende, entender las instituciones como un entramado de redes, es de vital importancia para una compleja comprensión desde el enfoque sistémico-complejo.
} 
por una parte su relación con los maestros entre sí, con el equipo directivo y con los psicólogos. Y, desde luego, el grupo de compañeros equivaldrá a la fratría 6 " (Linares, 2012, p. 108).

De esta manera, el sistema escuela es un constituyente de identidades que se sustenta desde diferentes autores que han aportado a la pedagogía como lo es por ejemplo Jean Piaget (1997), quien en su teoría del aprendizaje lo describe como un "proceso de organización de estructuras que se van asimilando y acomodando en las estructuras ya existentes causando desequilibrios cognitivos que se transforman de acuerdo con las experiencias vividas" (p.65). Luego, Vygotsky (2012) hace énfasis en la importancia de lo social y cultural para la adquisición de aprendizajes como fruto de un proceso colaborativo, con otros y en un ambiente, es decir la interacción como componente esencial de aprendizaje.

Bruner (2008) además plantea que la información que recibe el niño debe ser dotada de sentido para él y de esta manera logrará un aprendizaje significativo, de allí que se desglosan las escuelas constructivistas en las cuales el niño es partícipe activo de su conocimiento. El funcionamiento humano, según Bandura (1984) se explica en la reciprocidad tríadica, la cual se enfoca en que la conducta, los factores personales cognoscitivos $y$ los acontecimientos del entorno son determinantes en los procesos de enseñanza aprendizaje especialmente en la primera infancia.

Desde una perspectiva psicocultural de la educación, en la que distintos autores aportan a la construcción pedagógica de conocimiento, se encuentra Bruner (2008) quien dentro de sus postulados describe que:
La escuela juega un papel crítico en la formación del yo, no solo porque proporciona herramientas o habilidades fundamentales para la supervivencia social de los individuos sino porque es, después de la familia, el principal espacio de valoración y legitimación del yo, en otras palabras, es el espacio donde se experimenta la autoestima. No obstante, nuestra escuela es elitista, discriminadora, descalificadora y desconfirmante, sin hablar de otras plagas como racismo y el autoritarismo. Ninguna de ellas le hace bien a la autoestima y a la formación de una identidad yoica autónoma, crítica y propositiva (Bruner J, 2008, p. 29).

Por lo tanto, la escuela de hoy, y especialmente las instituciones educativas que atienden la primera infancia, tienen el gran reto de vencer concepciones academicistas, en las cuales se da prioridad a las construcciones de conocimiento o transmisión de saber a quienes no lo tienen: los niños y las niñas, deponiendo la importancia de las relaciones y lo afectivo, como parte esencial de una construcción de saberes. En esta misma línea, es necesario comprender que,

Los niños no aprenden por una relación lineal de causa-efecto ni por un tipo de enseñanza que realizamos de manera directa con ellos. Su aprendizaje es mérito, en gran parte, de los niños, de sus actividades y del uso de los recursos que poseen (Malaguzzi, 2011, p. 55)

Es decir, de unas habilidades y saberes que el niño trae consigo y pone en diálogo en otro espacio es lo que le permite o no seguir potenciando estas habilidades. Aquí, entra a jugar un rol fundamental el guía, en la medida que puede limitar o

${ }^{6}$ Sociedad íntima, hermandad, cofradía. Real Academia Española y Asociación de Academias de la Lengua Española (2014). 
Nutrición relacional: configuración de identidades como red sistémica entre familia e instituciones educativas en primera infancia.

favorecer el aprendizaje a través, de las relaciones e interacciones. Lo anterior entonces sugiere que la nutrición relacional en la escuela también emplea componentes cognitivos, pragmáticos y emocionales, por ejemplo, en lo cognitivo, la valoración y reconocimiento, que parte de promover un proceso autónomo y protagonista en la construcción de conocimiento del individuo. A partir de esto, surgen cuestionamientos de cómo el maestro está posicionando al niño, de qué manera se comunica con él, cómo corrige y cómo concibe el desarrollo socio-afectivo del mismo, en el que está involucrado, así como las familias y todo un equipo educativo que se encuentra en un espacio institucional determinado.

Como lo menciona Malaguzzi (2011), "lo que de verdad es importante es sembrar un terreno que haga emerger los acoplamientos estructurales entre lo cognitivo, lo relacional y lo afectivo" (p. 55) desde la llegada de las familias y niños a la institución como un espacio acogedor, de encuentros e interacciones constantes que nutra no solo al niño, niña sino además a las familias y todo un equipo educativo que forman parte de esa red sistémica en la que se configuran además identidades, en el tejido de relaciones e interacción de saberes que se va tejiendo para conformar narrativas.

De esta manera, la pedagogía relacional es una estrategia válida para un sistema educativo al comprender:

El sistema como una conjunción de elementos en interacción dinámica que tienen una finalidad común. Su fuerza se encuentra en poder expandir y cualificar las formas y los instrumentos relacional-interactivos que son los que mejor aseguran el flujo de las expectativas, actividades, intercambios, cooperaciones, conflictos, elec- ciones y el poder aclarar los problemas cognitivos, afectivos y expresivos. Entre sus objetivos está el poder reforzar en cada niño el sentido de su propia identidad a través del reconocimiento de los coetáneos y adultos, hasta que el niño pueda sentir ese grado de seguridad y de pertenencia que le permite aceptar y participar en la transformación de las situaciones que vive (Malaguzzi, 2011, p. 55).

En este sentido, dar fuerza a la idea de fomentar las redes comunicativas entre los individuos que confluyen en esta red sistémica: padres de familia, maestros, equipo educativo, niños y niñas, partiendo de los intercambios, los diálogos y la afectividad, que se proporcione en los primeros años de vida, seguramente serán las bases nutricias para la configuración de identidades que forjen una personalidad autónoma, segura, crítica, reflexiva y que además funciona como un potente transformador de las diferentes violencias que permean consciente e inconscientemente las prácticas pedagógicas en la familia e instituciones desde la primera infancia.

\section{Conclusiones}

Las reflexiones finales del presente artículo involucran un análisis desde la nutrición relacional como componente sistémico es indispensable en una red familia e instituciones educativas para la configuración de identidades de todos los individuos que hacen parte y que transforman una sociedad desde las primeras formas de relación e interacción que se puedan gestar en aquella red sistémica desde la primera infancia.

El enfoque sistémico, teorizado por Bertalanffy, constituye un modelo explicativo y relacional para la comprensión de realidades y configuración de identidades de niños y niñas de primera 
infancia, enmarcados en micro y macro sistemas que transforman y pluralizan la dimensión de aprendizajes conjuntos y relaciones entre maestros-padres-niños y niñas.

De esta manera, en un sistema donde las partes se relacionan con el todo y el todo con las partes, es indispensable pensarnos en una transformación desde las relaciones nutricias fundamentadas en el amor, en brindar ambientes de calidad para forjar una educación inicial que de paso a seres humanos para sí mismos, para los otros y para un mundo que es de todos. En este sentido, considerar la familia como un sistema, es entenderla como un conjunto con identidad propia y diferenciada, con una dinámica específica que se autorregula y mantiene procesos de cambio en su interacción con los macro sistemas en los que se encuentra. Además, permanece inmersa en un contexto social, cultural, político y económico cambiante, tal como las relaciones que se generan en su interior.

La nutrición relacional permanece entonces, dentro de procesos de configuración de identidades en los niños y las niñas, a partir, de la interacción de las dimensiones conyugal y paternal nutriéndose entre sí. Además, se evidencia desde la narrativa del individuo y construye formas de interactuar distintas.

Por otro lado, la escuela, por ser un espacio en el que transita la infancia desde sus primeros años de vida, debe encontrarse permeada de posibilidades en las que se generen relaciones, interacciones y conocimientos constantemente en cada uno de los momentos del día y espacios con todas las personas que están allí, que al mismo tiempo brinde oportunidades de aprendizaje especialmente en la socialización y desarrollo socioafectivo, determinantes para encaminar procesos constitutivos de la identidad de individuos para una sociedad.

Sin embargo, estos sistemas familia y escuela nombrados anteriormente no corresponden a ámbitos dependientes, sino interdependientes, como lo son los subsistemas conyugal y parental; de allí es entonces indispensable que exista una correspondencia, una conexión con cada uno de los sistemas que confluyen y nutren a los individuos niño-niña con las relaciones e interacciones que se generan entre ellos. De esta manera, la conexión entre familia y escuela, dentro de un trabajo conjunto y solidario por la configuración de identidades desde la infancia, que se nutran de percepciones de ser amados a partir el cuidado del ser, del otro y de lo otro, conforman una totalidad, un sistema capaz de nutrir a individuos en formación, en una red de relaciones coherentes y comprometidas, como totalidad a un contexto político, social, cultural y económico que transforme.

Maturana (2008) argumenta que es el amor aquella emoción que posibilita el lenguaje, es la emoción central en la historia evolutiva humana, el amor como la emoción que constituye el dominio de acciones en que nuestras interacciones recurrentes con otro hacen al otro un legítimo otro en la convivencia, es decir, rescata la importancia de las emociones en medio de un pensamiento constituido por paradigmas en donde la racionalidad tiene una valía superior y las emociones son la expresión simple del ser humano no pensante.

Nos encontramos. en el dilema entre el propósito individual y el propósito social, pues la necesidad de ser competitivo genera una negación del otro y por ende una pérdida de tejido social. En virtud de ello, es importante reconocer el educar como un proceso de transformación en la convivencia con otro, en ese espacio compartido, en esas 
Nutrición relacional: configuración de identidades como red sistémica entre familia e instituciones educativas en primera infancia.

relaciones de aceptación e interacción recíproca en el que es indispensable la reflexión. Actualmente, estos planteamientos difieren de una educación que estimula la competencia, la negación del otro, la apropiación y explotación del mundo natural antes que la coexistencia desde la aceptación legítima del mismo.

Es entonces el amor, las relaciones y emociones que se generan desde la primera infancia en un ambiente nutricio (familia - escuela), que aportan directamente a la configuración de identidad de los individuos. Teniendo en cuenta que estamos inmersos en y para una sociedad que debe reconocer a la primera infancia como un ciclo de vital indispensable, y siendo conscientes del compromiso en la transformación de las dinámicas relacionales para la configuración de una sociedad de la no violencia.

En este marco, se propone una nueva mirada a la educación como red sistémica familiainstituciones educativas para la primera infancia, pero además una visión holística en la cual se comprenda un macro sistema histórico, cultural, político, económico y social esencial en la configuración de identidades de los individuos inmersos en el mismo. Es también, replantear metodo- logías de enseñanza-aprendizaje basados en ideas especialmente enfocadas al desarrollo académico para poner énfasis en las relaciones socio-afectivas y extraordinarias capacidades de niños y niñas, durante sus primeros años de vida, que permita entender una nueva concepción sobre las identidades como un proceso de transformaciones $y$ reorganizaciones de manera permanente que convierten a las familias, pero en especial a ellas y ellos en individuos ejecutores, constructores de su conocimiento, capaces de comprender las ideas de los otros y de sí mismos.

Lo anterior, exige repensar el quehacer relacional con los niños y las niñas en su crianza y en la educación inicial. El llamado es a querer saber, descubrir y entender cómo son y cómo piensan, pero también como sienten, su potencial de involucrarse dentro del mundo, cómo sus redes más próximas (familia y escuela) les brindan las herramientas para fortalecerse y configurar una identidad que podría construirse desde las relaciones amorosas, coherentes y empáticas que transformen nuestra sociedad e individuos en seres relacionales, sociales, históricos que aporten a la misma y desdibujen poco a poco pautas culturales de violencia. 


\section{Referencias bibliográficas}

Ballester, L y Colom, A. (2012). Intervención sistémica en familias y en organizaciones socioeducativas. Barcelona, España: Octaedro.

Bandura, A. (1984). Teoría del aprendizaje social. Madrid, España: Editorial Calpe.

Bertalanffy, L. (1976). Teoría General de los Sistemas. Ciudad de México, México: Fondo de Cultura Económica.

Bourdieu, P, (2007). El sentido práctico. Argentina. Editorial: Siglo veintiuno.

Bronfenbrenner, U. (1986). Ecology of the family as a context for human development: research perspectives. Developmental Psychology, 22 (6), 723-742.

Bruner, J. (2008). Desarrollo cognitivo y educación. Madrid, España: Morata.

Cervantes, M. (1615). Don Quijote de la Mancha. Madrid, España: Alicante.

Espinal, I, Gimeno, A y González, (2000) F. El enfoque sistémico en los estudios sobre la familia. Universidad autónoma de Santo Domingo y centro cultural Poveda. España.

Fundación Carulla. (2015). Libro Horizontes, orientaciones pedagógicas y operativas para la implementación de la experiencia educativa aeioTU. Cartografía curricular, Fundación Carulla.

Greenspan, S. (2005). Las necesidades básicas de la infancia. Barcelona, España: Graó
Hernández, A. (1997). Familia, ciclo vital y psicoterapia sistémica breve. Bogotá, Colombia. Editorial El Búho.

Linares, J. L. (1996). Identidad y narrativa. La terapia familiar en la práctica clínica. Barcelona, España: Paidós.

Linares J \& carreras A, (2006). Diálogos sobre personalidad, identidad y narrativa. Recuperado de http://www.unizar.es/acaras/Tex.Dialogosidentidad.pdf

Linares, J. (2007). La personalidad y sus trastornos desde una perspectiva sistémica. Revista clínica y salud. 18 (3), 381-399.

Linares, J. L. (2012). Terapia Familiar Ultramoderna. La Inteligencia Terapéutica. Barcelona, España: Herder.

Malaguzzi, L. (2011). La educación infantil en Reggio Emilia. Madrid, España: Ed. Octaedro.

Martínez, C. (2013). En medio de una crisis de civilización: reflexiones sobre algunas evidencias. Bogotá, Colombia. Recuperado de 7096-Texto del artículo-32904-1-10-20140721

Maturana, H (1992) El sentido de lo humano. Santiago de Chile, Chile: Editorial Universitaria.

Maturana, H. (2008) Emociones y lenguaje en educación y política. Santiago de Chile, Chile: Colección Hachette/Comunicación.

Maturana, H y Varela, F. (2004) De máquinas y seres vivos. Autopoiesis: la organiza- 
Nutrición relacional: configuración de identidades como red sistémica entre familia e instituciones educativas en primera infancia.

ción de lo vivo. Santiago de Chile, Chile: Grupo Editorial Lumen.

Piaget, J. (1997). Psicología del niño. Madrid, España: Ediciones Morata.

Real Academia Española y Asociación de Academias de la Lengua Española (2014). Diccionario de la lengua española (23. ${ }^{\mathrm{a}}$ edición). Recuperado de https://dle.rae. es/?id=IQK7oMq

Sacristán, G. (2010) Educar y convivir en la cultura global. Madrid, España: Editorial Morata.
Vélez M. (2015) Comportamiento de la Violencia Intrafamiliar. Recuperado de http://www.medicinalegal.gov.co/documents/20143/49523/Violencia+intrafa miliar+primera+parte.pdf. Fundación Ideas para la $\mathrm{Paz}$

Vygotsky, L. (2012). Desarrollo de los procesos psicológicos superiores. Madrid, España: Austral.

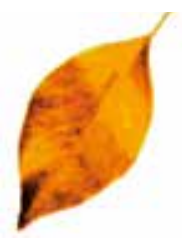

